\title{
Determination of tension of friction of piston of dispersible material is in a pipeline
}

\author{
Michail Vasilevsky ${ }^{1}$, Aleksandr Razva ${ }^{1 \mathrm{a}}$, and Yaroslav Grebenkov ${ }^{1}$ \\ ${ }^{1}$ National research Tomsk polytechnic university, 634050 Tomsk, Russia
}

\begin{abstract}
Estimates of the piston friction stress of particulate material in the pipe by mechanical action of the plunger. The material is compacted to form the roof and moves as a whole. The ranges of the coefficients of lateral pressure and coulomb friction.
\end{abstract}

\section{Introduction}

Necessity of moving of materials on considerable distances on the routes of difficult spatial geometry, portage with a high yield at the small rate of movement of dispersible environment resulted in development of the systems with the impulsive moving of material as pistons [1]. Pneumatic transport of bulk materials with a concentration close to the bulk, is more economical in comparison with other modes of transportation. At higher weight concentrations fullest energy carrier medium is used, there is no need to provide a speed at the beginning of weighing particle conduit, the rate of material exiting the pipe, the possibility is improved dust separation. However distance of transport the continuous stream at any overfall a $20 \mathrm{~m}$ is limited to [2]. With increase of distance a stream becomes unsteady: there is chaotic formation of pistons of friable material with the subsequent unevenness of their motion in relation to each other, by their association, destruction, and some cases corking of pipeline. In [3] the analysis of mechanisms of formation of breaks is conducted, to instability in the structures of the moved layers of material. To implement the extended-range transport is necessary to satisfy a number of difficult feasibility of actions, one of which is the use of stepwise expanding the pipeline and reduction of speed of rotation. It is concluded that the most effective during transportation over long distances is the use of a pulsed pneumatic piston, which through the use of small pressure differential across the portion of the granular medium pressure distribution close to the line and tensile forces are negligible [3-6].

\section{Theoretical bases}

By a basic obstacle under act of gas stream there is force of resistance of friction of material motion of pistons at the wall of pipeline. For the estimation of this force the hydrodynamic coefficient of friction of material is led at a wall, depending on the largeness of particles, closeness of particles, bulk mass, roughness of wall of pipe, attitudes of length of piston toward the diameter of pipe, concentration of particles in a stream $[1,2,6]$.

This coefficient is determined experimentally on the basis of model starts and measuring of parameters of streams in experimental options and industrial systems. In works [4-5] the coefficients of coulomb friction of different materials were determined at the wall of pipe in the moment of breakaway piston. It is found out that in case of occurring of overfall of pressure there is a compression of layer of the micronized material, in material there are irreversible deformations in back part of piston. The shift characteristics than the rate of increase of pressure, influenced by the ratio of length to diameter of the piston pipe, sealing material (packing density). In addition, a significant effect exerts pressure on the wall material by gravity, depending on the pipe diameter. In [7] it is shown that the movement of the piston exposed to the particulate filter flow through the piston wall shear stress is much smaller than the mechanical movement of the piston plunger.

\section{Research method and results}

With the device in Fig.1. studies have been conducted frictional stress at a wall of the bulk movement of the piston plunger. The aim of the study was to estimate the friction coefficient in the horizontal and vertical positions of the pipeline. In order to prevent entrapment of particles between the body of the plunger and the wall of the pipe was installed elastic insert, which created the initial shear strength.

\footnotetext{
${ }^{\text {a }}$ Corresponding author: razva@mail.ru
} 


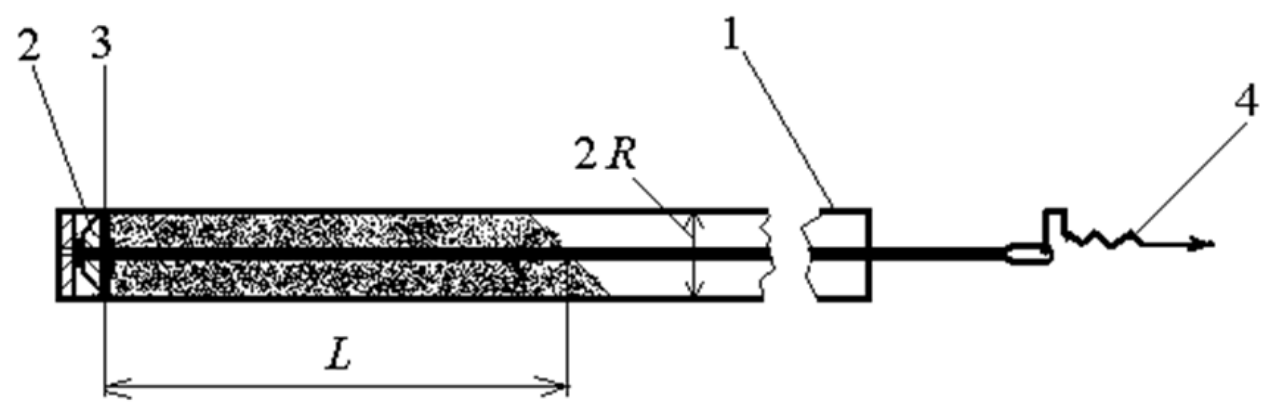

Figure. 1 Device for determination of tension of friction on the wall of piston friable: 1 - tube of $2 \mathrm{R}=21 \mathrm{~mm}$; 2- body plunger; 3 - elastic insertion; 4- dynamometer.

We used well dedusted bulk materials: stainless steel powder obtained dispersion melt, weathered sand, ordinary sand (river), granulated polypropylene, polished pearl barley. Characteristics of the materials shown in Table 1.

Table 1Characteristics of materials

\begin{tabular}{|c|c|c|c|c|}
\hline \multirow{2}{*}{ Name } & \multicolumn{3}{|c|}{ Parameters } & Note \\
\cline { 2 - 5 } & The angle of repose $\boldsymbol{\alpha}^{\circ}$ & Bulk density $\mathbf{~} \mathbf{H} \mathbf{g} / \mathbf{c m}^{\mathbf{3}}$ & Size $\mathbf{~ m m}$ & spheroid \\
\hline 1. Stainless steel & 22 & 4,71 & $0,1-1$ & spheroid \\
\hline 2. Sand tumbled & 33 & 1,78 & $0,1-1$ & angular \\
\hline 3. Sand ordinary & 34 & 1,57 & $0,1-1$ & cylinders F4, H3 \\
\hline 4. Granules polypropylene & 27 & 0,55 & $3-4$ & ellipsoids \\
\hline 5. Pearl barley & 32 & 0,82 & $3-6$ & \\
\hline
\end{tabular}

Fig. 2 shows the force values on the dynamometer according to the relative length of the piston in the vertical and horizontal

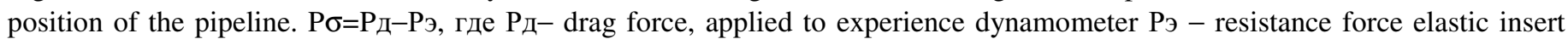
material $1 \mathrm{~cm}$ high $(\mathrm{L} / 2 \mathrm{R}=0,05)$ in the riser. The reproducibility of the results was $78 \%$.

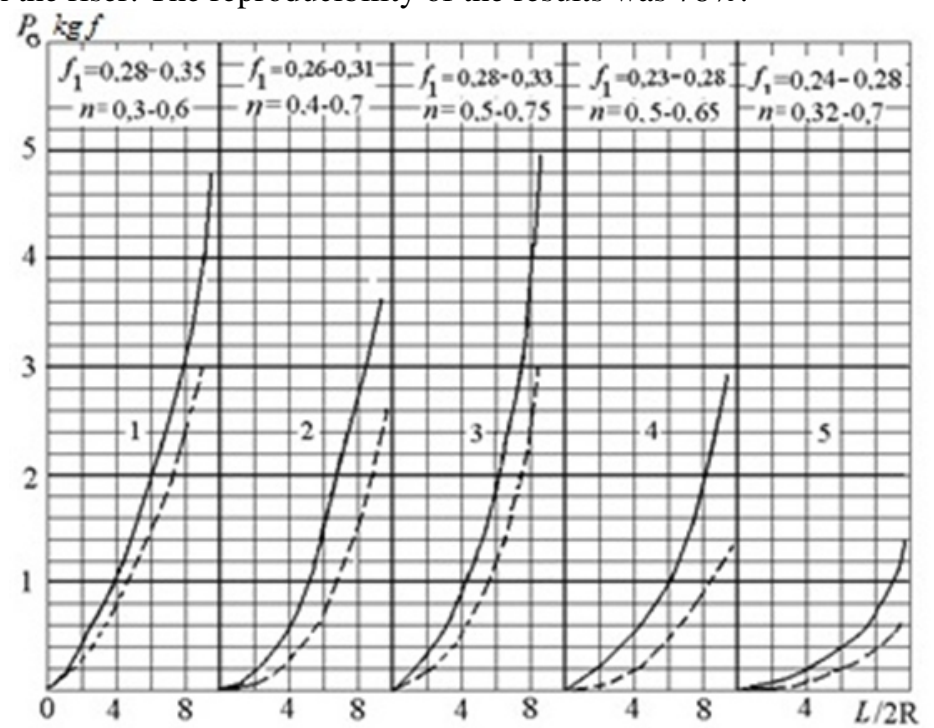

Figure 2. Dependence of effort of the plunger instead of resistance of elastic insertion from relative length of piston of dispersible material for a vertical (continuous curves) and horizontal pipeline (dotted curve). The numbers of curves correspond to the numbers of materials in a table

Both the external loading and adhesive contact is taken into account in the generalized law of friction [8]. Under the action of loading there is flattening of particles and growth of adhesive contact. For large particles an adhesive contact shows up in less degree, what for shallow. However with growth of pressure between contacting bodies arching is possible, that determines the feature of tensions of friction and coefficient of friction $[9,10]$.

On ris. 3 the chart of forces, operating on an element layer friable in a horizontal pipeline is presented. 


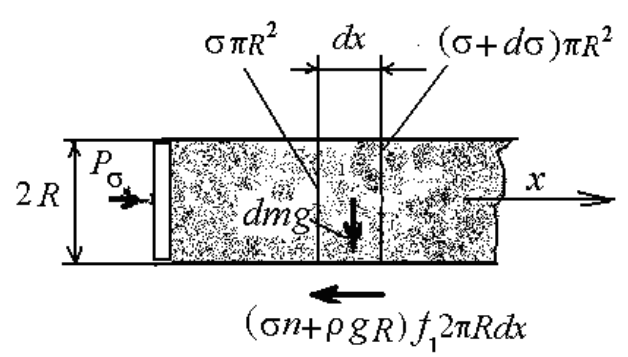

Figure 3. Driving forces on the element layer when the plunger.

The equilibrium equation element under the influence of forces has the form

$$
-d \sigma-(\sigma n+\rho g R) \frac{2 f_{1}}{R} d x=0
$$

Where $\sigma$ - the average cross section of the axial stress, $\rho$ - density layer in the element, $n$ - lateral pressure coefficient, $R$-radius pipeline, $f_{1}-$ coefficient of friction. Integrating equation (1) within $\sigma-0$ and $\mathrm{x}-\mathrm{L}$, assuming a constant $f_{1}$ and $\mathrm{n}$, we obtain the following expression of the distribution of axial stress in a horizontal pipe.

$$
\sigma_{\text {г }}=\frac{\rho g R}{n}\left[\exp \frac{2 f_{1} n(L-x)}{R}-1\right]
$$

For vertical piping solution for the distribution of axial stress is known [11]

$$
\sigma_{\text {в }}=\frac{\rho g R}{2 n f_{1}}\left[\exp \frac{2 f_{1} n(L-x)}{R}-1\right]
$$

When $f_{1} \rightarrow \sigma_{\Gamma} \rightarrow, \sigma_{\mathrm{B}} \rightarrow \mathrm{g}(\mathrm{L}-\mathrm{x})$. Introducing the mean stress over the length of the piston, $\bar{\sigma}_{\mathrm{\Gamma}}=\frac{1}{L} \int_{0}^{\mathrm{L}} \sigma_{\mathrm{\Gamma}} d x$ we obtain

$$
\bar{\sigma}_{\text {г }}=\frac{\rho g R}{n}\left\{\left[\frac{R}{2 f_{1} n L} \exp \left(\frac{2 f_{1} n L}{R}\right)-1\right]-1\right\}, \bar{\sigma}_{\mathrm{B}}=\frac{\rho g R}{2 f_{1} n}\left\{\left[\frac{R}{2 f_{1} n L} \exp \left(\frac{2 f_{1} n L}{R}\right)-1\right]-1\right\} .
$$

Average voltage determined by the relations of friction $\bar{\tau}_{\mathrm{r}}=n f_{1} \frac{R}{2 L} \bar{\sigma}_{\mathrm{\Gamma}} \bar{\tau}_{\mathrm{B}}=n f_{1} \frac{R}{2 L} \bar{\sigma}_{\mathrm{B}}$

Taking into account correlations (4), will get

$$
\bar{\tau}_{\mathrm{\Gamma}} / \bar{\tau}_{\mathrm{B}}=2 f_{1}=P_{\sigma \mathrm{\sigma}} / P_{\sigma \mathrm{B}}
$$

Equation (5) allows using curves Fig. 2, an evaluation of the friction coefficient, and the relation (2) - (4) - assessment values of the coefficient $n$.

The values nf1 found from expression (3) as $\mathrm{x} \rightarrow$, the values of n, f1 shown in Fig. 2.

\section{Conclusion}

The values obtained for the lateral pressure above 1.5 - 2 times higher than a calculation according to known formulas. The values of the coefficients of friction and lateral pressure depends on the length of the piston and the efforts on the plunger

\section{References}

1. Schwab V.A. Aeromechanical methods in the technology of production of powder . - Tomsk : Publishing house of Tomsk . University Press, 1984. - 161 p. 
2. Velshof G. pneumatic transport at high concentrations of particles of material moved . Trans. with it. - M .: Kolos.-

3. A.T. Sevastyanov The appearance and ways to eliminate porshneobrazovaniya in pneumatic dense layer. // Problems of heat and mass transfer and application aerohydromechanic Tomsk Tomsk Izd-vo . un-ta , 1983, p . 69-74.

4. Voronin V.N. Investigation of the stability of dispersed flow piston structure with respect to the pneumatic conveying systems: the thesis abstract on competition of a scientific degree of candidate of physical and mathematical sciences / V.N. Voronin ; Tomsk State University . -Tomsk : TSU Publishing House , 1996. -19

5. Voronin V.N., Egorov, V.M., Experimental study of friction features fine granular materials // Questions aerohydromechanic and heat and mass transfer. - Tomsk Tomsk Izd-vo. un-ta 1983: 85-91.

6. Klinzing G.E., Risk F., Marcus R., Leung L.S. Pneumatic Conveying of Solid- Springer, 2010.

7. M. Wasilewski, Romandin V.I., Zykov E.G. Assessment of the dispersion medium with particles of incoherent to install pneumatic piston // Proceedings of the All-Russian eighteenth. scientific and engineering. Conference "Energy: efficiency, reliability, safety." - Tomsk: Publishing house TPU, - 2012. C. 154-158.

8. Simon A.D. Adhesion of dust and powders. - M .: Chemistry, 1976. - 432 p.

9. Dzhenika E.V. Storage and release granular materialov._Per . from English. / ed. M.I. Agashkova -M .: "Peace », - 1968162 .

10. Ostrovsky G.M. pneumatic transport of bulk materials in the chemical industry / G.M. Ostrovsky . -L . : Chemistry, 1984. 104 pp.

11. R.L. Zenkov Mechanics of bulk cargoes . -M .: Engineering , 1964. - 251 . 